\title{
Cambios epidemiológicos y actualidades sobre vacunación contra Bordetella pertussis en Latinoamérica
}

\section{Epidemiologic changes and novelties on vaccination against Bordetella pertussis in Latin America}

\author{
Ángela Gentile1,2, Juan Pablo Torres Torreti1,3, Pío López López ${ }^{1,4}$ y Rolando Ulloa-Gutierrez ${ }^{1,5}$
}

Una versión en inglés de este artículo se publica en páginas: 243-253

${ }^{1}$ Sociedad Latinoamericana de Infectología Pediátrica (SLIPE).

Departamento de Epidemiología, Hospital de Niños "Dr. Ricardo Gutiérrez," y Universidad de Buenos Aires. Buenos Aires, Argentina.

${ }^{3}$ Departamento de Pediatría y Cirugía Infantil Oriente; Unidad de Infectología, Hospital Luis Calvo Mackenna; Facultad de Medicina, Universidad de Chile. Santiago, Chile.

${ }^{4}$ Departamento de Pediatría y Programa de Infectología Pediátrica, Universidad del Valle. Cali, Colombia.

5Servicio de Infectología, Hospital Nacional de Niños "Dr. Carlos Sáenz Herrera"; San José, Costa Rica.

Los autores declaran ausencia de conflictos de interés.

Sanofi facilitó la II Reunión de Expertos Latinoamericanos en Vacunación del Lactante, que sirvió como acercamiento de los autores para la preparación de este manuscrito. Sanofi no tuvo participación en el contenido ni en las conclusiones del manuscrito, que son responsabilidad exclusiva de los autores.

Recibido: 20 de noviembre de 2020 / Aceptado: 5 de febrero de 2021

\section{Resumen}

A pesar de que la tosferina (coqueluche) es una enfermedad prevenible por vacunas (EPV), la epidemiología latinoamericana muestra que hay persistencia de brotes en la región. Esta persistencia se debe, al menos en parte, a factores tales como la cobertura vacunal, la presencia de movimientos anti vacunas, la diversidad de los sistemas locales de vigilancia y la falta de una definición de caso unificada para la región. Dada la importancia de la tosferina en Latinoamérica y los cambios ocurridos en las recomendaciones para la vacunación, este manuscrito tiene como objetivo revisar los datos epidemiológicos y los cambios recientes en los calendarios de vacunación y su impacto sobre la enfermedad pediátrica por Bordetella pertussis en Latinoamérica. Los datos epidemiológicos más recientes muestran que entre regiones, países, y segmentos dentro de cada país hay heterogeneidad en la cobertura vacunal, con distintos rebrotes. Esfuerzos en la región han tratado de mejorar esta situación al introducir vacunas acelulares (aP), menos reactogénicas que las vacunas de células enteras (wP) en los calendarios vacunales. Además, algunos países han mejorado la definición de caso confirmado, al introducir la reacción de polimerasa en cadena (RPC) como criterio diagnóstico. En respuesta a las heterogeneidades de cada país y a la epidemiología actual de la región, un Comité de Expertos de la Sociedad Latinoamericana de Infectología Pediátrica (SLIPE) y la Asociación Latinoamericana de Pediatría (ALAPE) propone una definición unificada de caso y recomendaciones para mejorar la cobertura vacunal y reducir los brotes de tosferina en Latinoamérica.

Palabras clave: Bordetella pertussis; tosferina; coqueluche; vacunación; Latinoamérica.

\section{Abstract}

Although whooping cough is a vaccine-preventable disease (VPD), its epidemiologic characteristics in Latin America shows persistence of outbreaks in the region. This persistence is due, at least in part, to the presence of antivaccine movements, the diversity of the surveillance systems, and the lack of a uniform case definition for the region. Given the importance of whooping cough in Latin America and the changes in vaccine recommendations, this manuscript aims to review epidemiologic data and recent changes in the vaccination calendars and their impact on the pediatric disease by Bordetella pertussis in Latin America. Recent epidemiological data reveal that between regions, countries, and administrative units within each country there is a marked heterogeneity of vaccine coverage, with different outbreak patterns. Efforts in the region have tried to improve this situation by introducing acellular pertussis vaccines $(\mathrm{aP})$ in the vaccine calendars, which are less reactogenic than whole-cell pertussis vaccines (wP). Moreover, some countries have improved the case definition. Some countries have implemented a confirmed case definition by introducing polymerase chain reaction (PCR) as a diagnostic criterion. As a response to the heterogeneities observed within and between countries and the regional epidemiologic profiles, a Steering Committee from the Latin American Society for Pediatric Infectiology (SLIPE) and the Latin American Association of Pediatrics (ALAPE) propose a unified case definition and recommendations to improve vaccine coverage and reduce the outbreaks of whooping cough in Latin America.

Keywords: Bordetella pertussis; whooping cough; pertussis; vaccination; Latin America. 
Dada la importancia de la enfermedad por B. pertussis en Latinoamérica y los cambios ocurridos en las recomendaciones para la vacunación, este manuscrito tiene como objetivo revisar los datos epidemiológicos y los cambios recientes en los calendarios de vacunación y su impacto sobre la enfermedad pediátrica por $B$. pertussis en Latinoamérica.

do. Desde la década de los treinta se desarrolló una v celular completa (wP, por sus siglas en inglés para whole cell pertussis) en combinación con los toxoides contra difteria y tétanos (DTwP). La cobertura masiva mundial con DTwP redujo la tasa de infección (por 100.000 habs.) de 157 en la era pre-vacunación a menos de 1 en los años setenta ${ }^{1}$. Reportes sobre la reactogenicidad mediada por las toxinas de $B$. pertusis dieron pie al desarrollo de una vacuna acelular contra tosferina $(\mathrm{aP})$ en combinación con difteria y tétanos (DTaP para niños menores de 7 años de edad y Tdap para mayores de 7 años, esta última con menos concentración de los antígenos contra difteria y tosferina). Ensayos en la década de los noventa demostraron que las vacunas combinadas DTaP son mucho menos reactogénicas que las vacunas $\mathrm{DTwP}^{1}$. Sin embargo, pese al uso de vacuna, en la gran mayoría de los países persisten brotes o aumento en el número de casos cada 3 a 5 años $^{2}$.

Tres estudios recientes han recopilado y resumido el estado de vacunación contra tosferina en Latinoamérica $^{3-5}$. Estas revisiones mencionaron algunos desafíos para la región: diferencias inherentes a los sistemas de información, la falta de definiciones de caso normalizadas y la vigilancia epidemiológica de eventos adversos de naturaleza pasiva luego de la vacunación ${ }^{4,5}$. Además, se reportó que la poca representación geográfica limita la generalización de resultados y que la heterogeneidad de los métodos de los estudios limitan la comparación de resultados ${ }^{3}$.

La Sociedad Latinoamericana de Infectología Pediátrica (SLIPE) y la Asociación Latinoamericana de Pediatría (ALAPE) han adoptado un papel proactivo en cuanto a las recomendaciones para la vacunación contra $B$. pertussis. En una publicación conjunta reciente, ALAPE y SLIPE analizaron la continuidad de los servicios de salud más vulnerados por la pandemia en la región, en particular los de vacunación, e hicieron recomendaciones con base en las mejores prácticas reportadas en los países latinoamericanos ${ }^{6}$.

En Latinoamérica, la incidencia de la enfermedad por $B$. pertussis antes del año 2000 se estimó en 17,8 (intervalo de confianza [IC] al 95\% de 5,9 a 29,7) casos por 100.000 habs., mientras que a partir del año 2000 se estimó una incidencia entre 2,5 y 8,5 casos por 100.000 habs. ${ }^{3,4}$, con $90 \%$ de los casos reportados en lactantes bajo 1 año de edad ${ }^{4}$. La razón de fatalidad de los casos de tosferina en Latinoamérica se estimó en 3,9\% en la población general ${ }^{3}$ y en $4,8 \%$ bajo 18 años de edad ${ }^{4}$, aunque nuevamente, la mayoría de los casos se presenta en niños demasiado pequeños para ser vacunados ${ }^{4}$.

\section{Características epidemiológicas de la enfermedad por $B$. pertussis}

La tosferina es una enfermedad respiratoria endémica de distribución global con ciclos de brotes cada 3-5 años $^{7-10}$. A pesar de ser una EPV, la tosferina tiene altas tasas globales de hospitalización, complicaciones y muertes, especialmente en lactantes bajo 2 meses de edad ${ }^{7-11}$. A pesar del aumento global de las coberturas vacunales contra tosferina, durante 2018 se notificaron 151.000 casos en el mundo ${ }^{10}$.

Para disminuir su transmisión, la cobertura vacunal debe ser superior a $90 \%$ y, para ser efectiva, debe ser homogénea dentro de un país y entre países de la región?. En caso contrario, aparecen brotes epidémicos que afectan prioritariamente a los grupos más vulnerables, especialmente los lactantes bajo 1 año de edad.

La tosferina está subnotificada ${ }^{9}$, lo cual se debe fundamentalmente a las variaciones regionales en la definición de caso y a los distintos métodos diagnósticos utilizados en cada país, especialmente con la incorporación de la reacción de polimerasa en cadena (RPC), que aumenta significativamente la sensibilidad para la detección de B. pertussis ${ }^{12,13}$. Por ende, homogeneizar esta definición para la región permitirá una vigilancia epidemiológica adecuada y comparable entre países y regiones. En una reunión reciente, un grupo de trabajo de expertos latinoamericanos y de la Organización Panamericana de la Salud (OPS) propuso una definición homogénea de casos (Tabla 1) $)^{14}$.

Es importante mencionar que no todas las personas infectadas cumplen con todos los criterios para la definición de caso. Se pueden presentar pacientes- generalmente adolescentes y adultos-asintomáticos o con síntomas leves, tales como tos persistente, que no presentan otros síntomas y no tienen una carga importante de morbilidad directa. Dado que la enfermedad tiene un número reproductivo $\left(R_{0}\right)$ entre 15 y 17, se considera altamente contagiosa ${ }^{15}$. Los casos atípicos particularmente ponen en riesgo a los niños más pequeños no vacunados o con esquemas vacunales incompletos. Una revisión reciente de 16 estudios encontró que la tasa de ataque secundario es mucho más marcada en lactantes $(70 \%)$ que en el resto de la población $(20 \%)$ o en mayores de 40 años $(6 \%)^{16}$. Esta revisión también encontró que $42,6 \%$ de los contactos do- 
Tabla 1. Definición de caso unificada recomendada por el Grupo de Expertos para los casos probables, sospechosos, confirmados y descartados de tosferina

\section{Caso probable}

Paciente con clínica compatible con tosferina y sin confirmación por laboratorio (no estudiado o resultados de laboratorio No conclusivos) y sin nexo epidemiológico con un caso confirmado por laboratorio

$$
\begin{aligned}
& \text { Caso sospechoso } \\
& \text { Menores de } 6 \text { meses } \\
& \text { Toda infección respiratoria aguda, } \\
& \text { MÁS } \geq 1 \text { de los siguientes síntomas: } \\
& \text { - Apnea } \\
& \text { - Cianosis } \\
& \text { - Estridor inspiratorio } \\
& \text { - Vómitos después de toser } \\
& \text { - Tos paroxística }
\end{aligned}
$$

Paciente con clínica incompleta o datos insuficientes y con resultado positivo por RPC o por seroconversión
Mayores de 6 meses hasta los 11 años Tos de $\geq 14$ días de duración sin otra causa aparente, MÁS $\geq 1$ de los siguientes síntomas:

- Tos paroxística

- Estridor inspiratorio

- Vómitos después de toser

\section{Caso confirmado}

Cultivo positivo por B. pertussis MÁS cualquier infección respiratoria

Reacción de polimerasa en cadena (RPC) positiva

MÁS clínica compatible según la definición de caso sospechoso

Seroconversión

MÁs clínica compatible según la

definición de caso sospechoso

Nexo epidemiológico con caso confirmado mediante laboratorio MÁs clínica compatible según la definición de caso sospechoso

\section{Caso descartado}

Clínica incompleta o datos insuficientes, y con resultado de laboratorio negativo y sin nexo epidemiológico con un caso confirmado

Mayores de 11 años

Tos persistente de $\geq 14$ días de

duración, sin otra sintomatología

acompañante

Fuente: Modificado de la referencia 14.

mésticos se presenta con tosferina leve o atípica y 55,6\% de los contactos estudiados tenían infección asintomática documentada por laboratorio.

\section{Epidemiología actual de tosferina en Latinoamérica}

Datos recientes de la OPS, OMS, y UNICEF muestran una tasa de incidencia importante en el lactante bajo 1 año

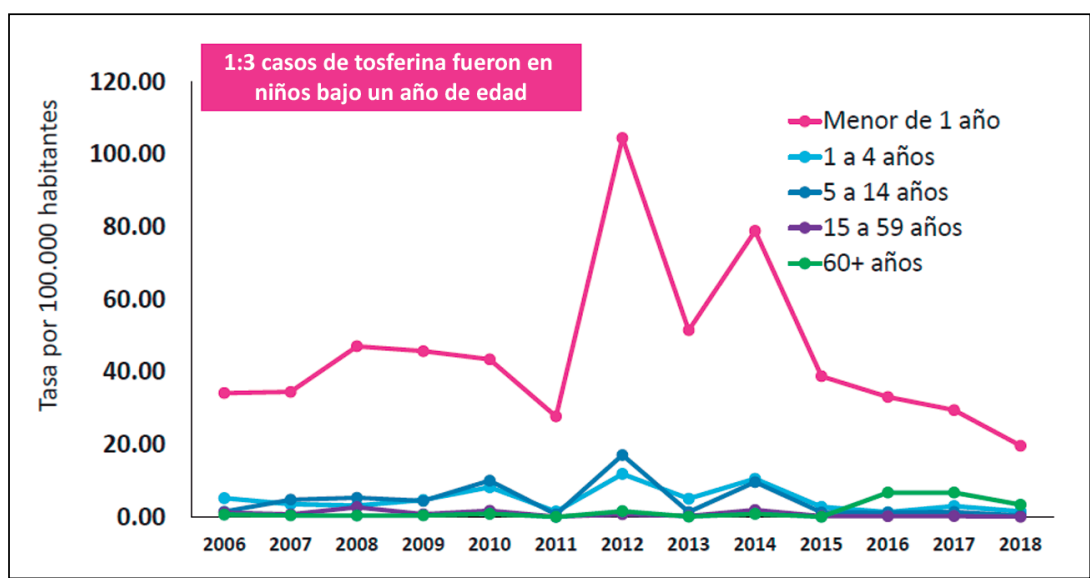

Figura 1. Tasa de incidencia de tosferina en Latinoamérica, 2006-2018. Fuente: Datos 2006-2018 del formulario conjunto para la notificación sobre inmunización de la OPS/OMS y UNICEF, 2019 (JRF, por sus siglas en inglés). de edad y que los grupos de 1 a 4 años y adolescentes y adultos jóvenes también se deben tener en cuenta (Figura 1). Por ello, las incidencias deben registrarse según grupos de edad, a través de un programa de vigilancia activa, especialmente en el grupo de adolescentes y adultos jóvenes.

A continuación, revisamos la situación 2018-2020 de algunos países de la región latinoamericana, que se resumen en la Figura 2. Cabe destacar que estos datos presentan limitantes previamente descritas ${ }^{3-5}$, que incluyen heterogeneidad entre los sistemas de información, falta de definiciones de caso normalizadas y vigilancia epidemiológica pasiva, con poca representación geográfica, especialmente de países más pequeños o menos populosos $^{3-5}$.

\section{Argentina}

Argentina presentó un pico de incidencia de tosferina en el 2012, con registro de más de 60 muertes, la mayoría en lactantes bajo 3 meses de edad. Eso llevó a la recomendación nacional para la inmunización universal de las mujeres embarazadas con Tdap. En los años siguientes (2013-2019), la enfermedad tuvo una incidencia menor que en años previos, al igual que en la región, pero presentó mayor incidencia en los niños bajo 1 año de edad. Se observó también una reducción de coberturas de vacunación incluso antes de la pandemia de COVID19. 
Perú, 2020

Perú, 2019

Perú, 2018

México, 2019

México, 2018

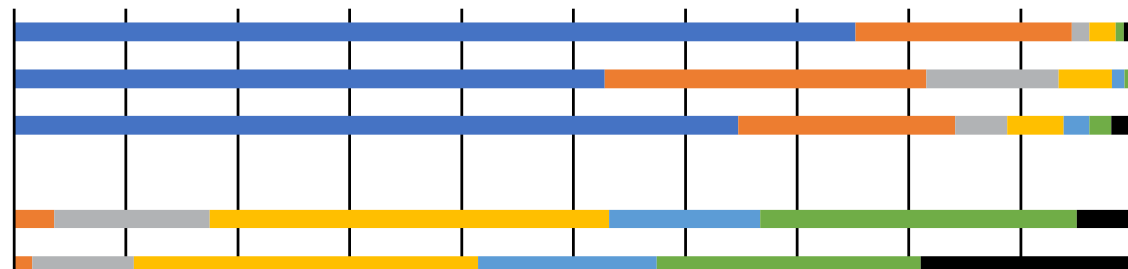

Costa Rica, 2019

Colombia, 2019

Chile, 2019

Brasil, 2020

Brasil, 2019

Brasil, 2018

Argentina, 2019
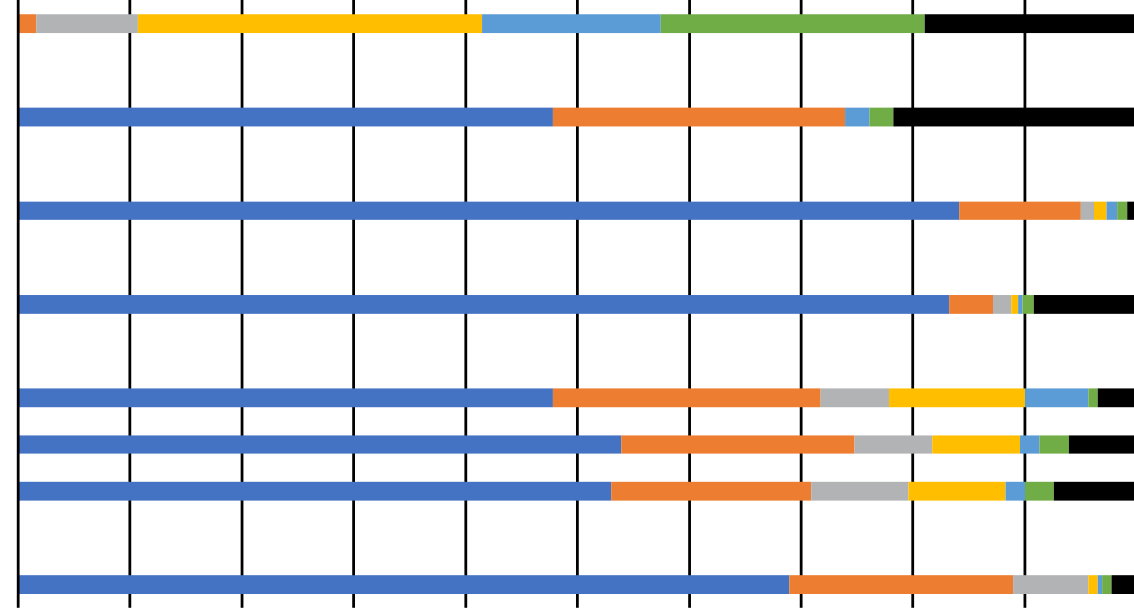

.

10

20

30

$40 \quad 50 \quad 60$

70

80

90

100

$\square<1$ año $\square$ 1-4 años $\square$ 5-9 años $\square$ 10-14 años $\square$ 15-19 años $\square$ 20-29 años $\square 29$ años

Figura 2. Distribución por edad de los casos de tosferina en países selectos de Latinoamérica, 2018-2020. Notas: México reportó los grupos de edad en incrementos de 3 años y se ajustaron a los grupos que mejor corresponden en la clasificación presentada. Costa Rica y Colombia presentaron datos del primer semestre de 2019. Fuentes: Argentina: Dirección de Control de Enfermedades Inmunoprevenibles en la base de datos SNVS 2.0. Ministerio de Salud. Brasil: SINAN, SVS/MS, al 25 de enero de 2020, obtenidos a través del Sistema Electrónico de Servicio e Información al Ciudadano el 03/03/2020. Chile: Base de datos ENO y base de datos EPIVIGILA al 10 de enero de 2020. Departamento de Epidemiología, DIPLAS. Ministerio de Salud. Colombia: Instituto Nacional de Salud, Ministerio de Salud (Colombia). Tos ferina: Colombia primer semestre de 2019. Bogotá, Colombia: 31 de mayo de 2019. Documento FOR-R02.4000-001. Costa Rica: Chanto, Grettel. Alerta: Aumento de casos de tosferina, Costa Rica, enero-febrero 2019. Tres Ríos, Costa Rica: INCIENSA, 2019. Consultado el 06 de octubre de 2020 de https://www.inciensa.sa.cr/actualidad/Alertas/2019/Alerta\%20aumento\%20de\%20casos\%20de\%20tosferina,\%20 Costa\%20Rica\%20enero-febrero\%202019.pdf. México: Boletín de Vigilancia Epidemiológica. Secretaría de Salud de México. Perú: Centro Nacional de Epidemiología, Prevención y Control de Enfermedades. Ministerio de Salud.

\section{Brasil}

En Brasil, la incidencia de tosferina es muy alta en lactantes, y aumentó dramáticamente entre 2013 y 2014, cuando se registraron más de 8.000 casos y más de 100 defunciones, la mayoría en lactantes bajo 3 meses. En 2014, el calendario nacional introdujo la vacuna Tdap para mujeres embarazadas y profesionales de salud.

Es de notar que en los últimos años hay una tendencia al aumento de casos en el grupo de adolescentes de 10 a 14 años, que puede estar asociada a la introducción de la RPC en centros de referencia y a la mayor concienciación de los profesionales de salud sobre la enfermedad después del brote más reciente.

\section{Chile}

Presenta características cíclicas con un pico en el año 2012. Los datos por edad de 2019 muestran que los lactantes bajo 1 año constituyen el grupo de mayor riesgo, al igual que en toda Latinoamérica. En Chile, el diagnóstico se confirma mediante RPC. Sin embargo, cabe notar que, en los adolescentes con más de 14 días de tos, la RPC pierde sensibilidad diagnóstica y requiere de dos muestras (RPC y serología), lo cual dificulta el diagnóstico de los adolescentes en los laboratorios de referencia. La experiencia de Chile con la RPC nos apunta a que en América Latina debemos trabajar en conjunto para hacer de la RPC el estándar diagnóstico. Así, los laboratorios de la región 
podrían estar en red para la implementación y validación de la tecnología y para proveer un diagnóstico oportuno y mejorar la comparabilidad de tasas entre países.

\section{México}

En el 2007, México introdujo la vacunación con aP en una sola vacuna pentavalente y en 2012-2013 incorporó la RPC como estándar diagnóstico. Se encontró que inmediatamente después de estos cambios hubo un aumento de casos reportados, que se explica por el cambio en la metodología diagnóstica que incorpora una nueva definición de caso y dificulta la comparación adecuada de incidencia con la serie histórica. En el 2019 hubo aumento de casos en niño vacunados con vacuna aP pentavalente, pero también en adolescentes y adultos jóvenes (17-24 años), vacunados previamente con wP. Esto revela que el aumento de casos observado en México no está relacionado al tipo de vacuna utilizada, y que el sistema de vigilancia no se debe enfocar exclusivamente en niños. La tosferina afecta a personas de todas las edades y, por ende, no sólo el pediatra debe estar vigilante.

\section{Perú}

También se observan características cíclicas y una tendencia a la disminución de los casos. Nuevamente, los lactantes bajo 1 año contribuyen con $75 \%$ de los casos, para una incidencia acumulada de 0,40 por cada 100.000 habs.

\section{Vacunación contra B. pertussis}

Existen dos tipos de vacunas contra $B$. pertussis: las vacunas de células enteras (wP) y las vacunas acelulares (aP). Las vacunas wP utilizan la bacteria entera, que puede ser de distintas cepas, inactivada por métodos físicos o químicos. Por ende, contienen muchos antígenos y tienen inmunogenicidad, eficacia y patrón de seguridad variables, que presentaremos en esta revisión.

Con el desarrollo de vacunas aP, que contienen menor número de antígenos (de 1 a 5), están mejor reguladas y presentan menor reactogenicidad, los países desarrollados incorporaron las vacunas $\mathrm{DTaP}$ en sus programas de vacunación en formulaciones pentavalentes (con adición de Haemophilus influenzae b y hepatitis B) o hexavalentes (con la adición de polio inactivado [IPV]). Las vacunas wP continúan siendo utilizadas en los países en vías de desarrollo. La información sobre la inmunogenicidad, eficacia y seguridad de las vacunas wP utilizadas hoy en día es muy limitada ${ }^{9}$, pero discutiremos los datos más recientes al respecto.

Dentro de la región centroamericana, la mayoría de los países utilizan wP en sus esquemas nacionales, excepto Costa Rica, Panamá y México que ya incluyen aP para niños. En la región andina (Bolivia, Venezuela, Colombia, Perú) y la mayoría del cono sur (Argentina, Brasil, Paraguay, Uruguay) sólo se utiliza wP, siendo Chile la excepción al introducir la vacuna aP hexavalente desde el 2018. Muchos de los países de la región ya administran Tdap a mujeres embarazadas (Tabla 2).

La adopción de vacunas wP en la década de los cincuenta, tanto en Japón ${ }^{17}$ como en Suecia ${ }^{18}$, demostró una reducción en la tasa de incidencia de tosferina, con reportes de reactogenicidad, que podrían explicar, al menos en parte, la reticencia de la población a la vacunación y una disminución en la cobertura vacunal. A su vez, esta reducción en la cobertura se tradujo en un resurgimiento de tosferina que propulsó la introducción de vacunas aP (Japón en los setenta y Suecia en los noventa), con adecuado control posterior ${ }^{17,18}$.

Otra explicación para los resurgimientos es la reducción (waning) de la inmunidad ${ }^{19}$. Los países que han introducido vacunación, tanto aP como wP en distintos esquemas, muestran resurgimientos cíclicos de tosferina. Por estas razones, la planificación de la vacunación contra $B$. pertussis debe seguir el ciclo de la vida con indicaciones específicas para lactantes, adolescentes, adultos y mujeres embarazadas ${ }^{4}$.

\section{Reactogenicidad y efectividad de wP y aP}

No todas las vacunas wP son iguales. Un estudio en Irán demostró diferencias significativas en cuanto reactogenicidad local y sistémica tras la vacunación con DTwP producida localmente ${ }^{20}$. Las diferencias en relación con los eventos adversos e inmunogenicidad pueden estar asociadas a las cepas utilizadas en la producción de las vacunas, a los métodos de preparación o la formulación de las vacunas.

Un estudio comparó aP con wP, verificando que las vacunas acelulares tienen menos efectos adversos: menos fiebre, dolor y edema local en el sitio de la punción ${ }^{24}$. Además, los niños vacunados con aP presentan un menor riesgo de eventos adversos neurológicos, especialmente episodios de hipotonía-hiporrespuesta y convulsiones ${ }^{25}$. La experiencia canadiense tras introducción de aP en 1997-1998 mostró una reducción importante en los episodios de convulsión febril $(60 \%)$ y episodios de hipotonía-hiporrespuesta $(67 \%)^{26}$. Un análisis de los datos de 567.378 niños nacidos antes y después de la introducción de vacunas aP en Canadá, encontró una reducción significativa del riesgo relativo de acudir a un servicio de urgencias por efectos adversos frecuentes asociados a la vacunación con $\mathrm{wP}^{27}$. Esto tuvo 38 veces mayor impacto comparado con la reducción de eventos más raros como convulsiones ${ }^{27}$.

Diversos estudios han demostrado que las vacunas 


\begin{tabular}{|c|c|c|c|c|c|c|c|c|c|c|}
\hline País & $2 \mathrm{~m}$ & $4 \mathrm{~m}$ & $6 \mathrm{~m}$ & $12 \mathrm{~m}$ & $15 \mathrm{~m}$ & $18 \mathrm{~m}$ & $4 a$ & $6 a$ & $10 a$ & $\begin{array}{c}\text { Mujeres embarazadas } \\
\text { (Tdap) }\end{array}$ \\
\hline \multicolumn{11}{|c|}{ México y Centroamérica } \\
\hline Belice & WP5 & WP5 & WP5 & & & & WP3 & & & \\
\hline Costa Rica & aP5 & aP5 & aP5 & & aP5 & & $\mathrm{aP} 4$ & & & 20 sem \\
\hline El Salvador & wP5 & wP5 & wP5 & wP5 & & & wP3 & & & \\
\hline Guatemala & WP5 & WP5 & WP5 & & & wP3 & wP3 & & & $3^{\text {er }}$ trimestre \\
\hline Honduras & wP5 & wP5 & wP5 & & & wP3 & wP3 & & & 26 sem \\
\hline México & aP6 & aP6 & aP6 & & & aP6 & wP3 & & & 20 sem \\
\hline Nicaragua & wP5 & wP5 & wP5 & & & wP3 & & wP3 & & \\
\hline Panamá & aP6 & aP6 & aP6 & & & WP4 & wP3 & & aP3 & $3^{\text {er }}$ trimestre \\
\hline \multicolumn{11}{|l|}{ Zona Andina } \\
\hline Bolivia & WP5 & WP5 & WP5 & & & WP5 & WP5 & & & \\
\hline Colombia & WP5 & WP5 & WP5 & & & WP3 & WP3a & & & $3^{e r}$ trimestre \\
\hline Ecuador & wP5 & wP5 & wP5 & & & wP3 & wP3 ${ }^{a}$ & & & \\
\hline Perú & WP5 & WP5 & WP5 & & & WP3 & wP3 & & & $3^{\text {er }}$ trimestre \\
\hline Venezuela & wP5 & wP5 & wP5 & & & WP5 & WP5 $5^{a}$ & & & \\
\hline \multicolumn{11}{|l|}{ Cono Sur } \\
\hline Argentina & wP5 & wP5 & wP5 & & & wP5 & & wP3 & aP3 & 20 sem \\
\hline Brasil & WP5 & WP5 & WP5 & & WP3 & & WP3 & & & 20 sem \\
\hline Chile & aP6 & aP6 & aP6 & & & aP6 & & aP3 & aP3 ${ }^{b}$ & 28 sem \\
\hline Paraguay & WP5 & wP5 & wP5 & & & WP3 & wP3 & & aP3 & 20 sem \\
\hline Uruguay & wP5 & wP5 & wP5 & & wP5 & & wP3a & & aP3 & 28-36 sem \\
\hline
\end{tabular}

aP registradas tienen eficacia comprobada en niños, independientemente del número de componentes ${ }^{8,21-23}$.

Una revisión sistemática con meta-análisis y modelado matemático publicada recientemente ${ }^{28}$ describió la efectividad de las vacunas aP (EV) después de 5 dosis de la serie infantil (EV: $91 \%$; intervalo de confianza [IC] al 95\%: 87-95\%) y después de la sexta dosis en adolescentes (EV: 70\%; IC al 95\%: 54-86\%). El modelado matemático demostró una disminución anual de la EV luego de 5 y 6 dosis $(9,6 \text { y } 45,3 \% \text { al año, respectivamente })^{28}$. Luego del refuerzo, la EV absoluta se estimó en $85 \%$ (IC al 95\%: $84-86 \%$ ) con una disminución de $11,7 \%$ (IC al $95 \%$ : $11,1-12,3 \%$ ) anualmente hasta un $28,2 \%$ a los 18 años $^{28}$. El estudio concluyó que las vacunas aP tienen alta eficacia inicial que disminuye con el pasar del tiempo ${ }^{28}$. Esto es fundamental, ya que los estudios observacionales sobre EV fallan en reconocer que miden datos de eficacia relativa y no EV absoluta, como fue el caso del meta-análisis presentado. Es importante resaltar que ninguna vacuna, aP o wP, ni tampoco la infección natural por B. pertussis es capaz de conferir protección duradera.

En los Estados Unidos de América, el estudio del sistema Kaiser Permanente de California, que involucró a 469.982 niños entre 3 meses y 11 años, reportó 738 casos de tosferina, distribuidos así: 99 no estaban vacunados, 36 estaban sub-vacunados y 515 estaban completamente vacunados. Comparados con los niños completamente vacunados, los niños no vacunados tenían un riesgo 13 veces mayor de enfermar y los niños subvacunados tenían un riesgo 1,9 veces mayor $^{29}$. Los subanálisis por edad encontraron que en los niños de 19-84 meses, el riesgo aumenta cinco veces en vacunados mayores de 3 años comparados con los niños bajo 1 año y en los niños de 84-132 meses, el riesgo aumenta dos veces en vacunados mayores de 6 años comparados con los niños bajo 3 años.

Durante la transición de wP a aP en Turquía, se llevó a cabo un estudio prospectivo con más de 1.400 lactantes $^{30}$. El estudio encontró diferencias significativas en reacciones locales $(9,1$ vs. $0,9 \%)$, sistémicas (12 vs. $1 \%$ ) y fiebre (26 vs. $2,6 \%$ ). Los lactantes vacunados con DTaP a los 2 meses tuvieron más reacciones sistémicas $(1,9 \%)$ comparados con aquellos vacunados a los $4(0,92 \%)$, $6(0,6 \%)$ y 18 meses $(0,13 \%)$. La vacuna wP fue más reactogénica a todas las dosis ${ }^{30}$. 
El grupo de Patterson ${ }^{31}$ analizó 47 estudios, en su mayoría ensayos clínicos controlados, que reportaban eventos adversos tras la vacunación con wP o aP. El análisis encontró riesgo relativo (RR) significativamente mayor con wP en cuanto a reacción local (RR 2,73), dolor (RR 4,15) o edema (RR 4,38) en el sitio de inyección, fiebre (RR 9,21), somnolencia (RR 1,34) y vómitos (RR $1,28) .{ }^{31}$ Es posible que las autoridades de salud consideren los eventos adversos locales como mínimamente relevantes en la toma de decisiones en cuanto a vacunas. Sin embargo, este estudio demuestra que los eventos adversos locales pueden interferir con la aceptación de las vacunas y resultar en bajas coberturas o retraso en la vacunación infantil. Además, la OMS considera que la reducción del dolor local asociado con vacunas es una buena práctica de vacunación ${ }^{32}$.

Pese a las coberturas vacunales relativamente altas contra $B$. pertussis, la bacteria continúa circulando en la comunidad, ya que ni wP ni aP evitan la colonización faríngea.

\section{Experiencia con aP en Latinoamérica}

A continuación, presentamos la experiencia latinoamericana con los países que han introducido vacunación con aP en la región, en el orden en que han introducido la vacuna.

\section{Costa Rica}

En los años 2006 y 2007 fueron registrados brotes de tosferina con más de 2.000 casos y más de 20 muertes, nación que entonces utilizaba vacunas wP. El país decidió cambiar para una vacuna combinada pentavalente aP + IPV en 2010, observando desde entonces pocos casos de tosferina, en su mayoría en niños muy jóvenes, hijos de madres que no habían recibido la vacuna Tdap durante el embarazo y en poblaciones indígenas. La experiencia con la introducción de la vacuna pentavalente aP + IPV ha sido muy buena, observándose aumento en las coberturas y raros reportes de eventos adversos.

\section{México}

En 2007, México introdujo una vacuna pentavalente aP + IPV, después del registro de dos casos de poliomielitis en bebés vacunados con la vacuna oral. Poco tiempo después, registró un aumento el número de casos de tosferina, pero ese aumento estuvo asociado con la introducción de la RPC al diagnóstico de la enfermedad en 2013 y no con el cambio a la vacuna aP. Recientemente se publicó la experiencia mexicana tras analizar 192 casos confirmados de tosferina en lactantes bajo 1 año ${ }^{13}$. Del total de casos, 80 ocurrieron en lactantes bajo 2 meses, 79 entre los 2 y 4 meses, 22 entre los 4 y 6 meses y 11 entre los 6 y 12 meses. El estudio resalta que ningún caso ocurrió en niños que completaron la serie primaria de tres dosis y refuerza la importancia de vacunar contra tosferina con tres dosis de una vacuna aP durante el primer año de vida. En 2019, nuevamente se observó aumento de casos en comparación con 2018, pero ese aumento no fue observado solamente en niños, sino que también en adolescentes y adultos jóvenes. En México las madres fueron las principales transmisoras de $B$. pertussis a sus niños $(25,4 \%$ de 437 contactos epidemiológicos documentados $)^{13}$, y desde el año 2012, se introdujo la vacuna Tdap para mujeres embarazadas.

\section{Argentina}

En Argentina, la vacunación contra $B$. pertussis incluye un esquema primario (a los 2, 4 y 6 meses) y dos refuerzos con componente wP (a los 15-18 meses y a los 4-6 años), una dosis de Tdap a los 11 años y en cada embarazo, refuerzo con vacuna Tdap. Las coberturas en lactantes bajo 2 años resultan insatisfactorias especialmente en la dosis de refuerzo de los 15 a 18 meses $^{33}$. En contraposición, la vacunación materna muestra coberturas en ascenso desde su recomendación en 2012 y su posterior incorporación al calendario en 2014. Las mujeres embarazadas se vacunan con Tdap a partir de las 20 semanas de gestación en todos los embarazos, independientemente de la edad, intervalo inter-genésico y del antecedente de vacunación con componente antitetánico. Después de la incorporación de la vacunación materna con Tdap, las tasas de letalidad muestran una tendencia en descenso sostenido a pesar de los cambios en la incidencia de la enfermedad ${ }^{34,35}$. Los trabajos demostraron una efectividad con Tdap de $80,7 \%$ (intervalo de confianza al 95\%: 52,1-92,2\%) en niños bajo 2 meses. Se halló similar efectividad cuando se administró Tdap durante el segundo o tercer trimestre ${ }^{34,35}$.

\section{Brasil}

Hasta 2014, en Brasil, se utilizó una vacuna wP tetravalente (DTwP + Hib) producida localmente, cuando se sustituyó por la vacuna pentavalente en el esquema primario. La serie primaria alcanzó $95 \%$ de cobertura, pero tales coberturas no eran homogéneas dentro del país, lo que conllevó al registró de brotes en diferentes regiones. En vista de lo anterior (ver párrafo sobre epidemiología de la tosferina en Brasil, pág. 235), el año 2014 se introdujo la vacuna Tdap para mujeres embarazadas, observándose gran reducción en el número de casos, a pesar de la reducción en las coberturas a partir de 2016. En julio de 2019, una vacuna pentavalente con componente wP producida en el Laboratorio Biologicals E Ltd., fue rechazada por las autoridades regulatorias brasileñas (Agência Nacional de Vigilância Sanitária [ANVISA]) por un problema de calidad, con la conse- 
Américas las coberturas para la serie primaria de tres dosis (DTP3) estuvieron cerca de 90\% en el año 2018, pero pocos países presentan coberturas altas y homogéneas, observándose enormes variaciones regionales en las coberturas de la serie primaria entre países (provincias, estados, municipalidades o distritos). Es importante destacar que la cobertura vacunal es un indicador "duro" que no permite identificar el riesgo para los niños con esquemas de vacunación atrasados. Por ende, se deben desarrollar indicadores que tomen en cuenta el impacto sobre las poblaciones pediátricas. Por otra parte, la cobertura con cuatro dosis completadas de difteria, tosferina y tétanos (DPT4) comparada con la cobertura con tres dosis (DPT3) muestra que la DPT4 está por debajo del $80 \%$ óptimo y que la cobertura no es homogénea para la región (Figura 3).

A pesar de la persistencia de factores que afectan la cobertura vacunal contra tosferina en Latinoamérica (e.g., movimientos antivacunas, reticencia de la población, adaptación de las cepas a través de cambios antigénicos, reemergencia y descenso de la inmunidad con el tiempo), generalmente se acepta que la vacunación contra la tosferina es segura y eficaz. El mayor impacto se observa en el lactante bajo 1 año que recibe la serie completa, reduciendo la carga de la enfermedad. Sin embargo, las coberturas vacunales deben ser altas y homogéneas para lograr una verdadera disminución en la incidencia de la enfermedad.

La reemergencia de tosferina se observa de la mano con la redefinición de casos con el uso diagnóstico de la RPC, incluyendo las formas atípicas que no se diagnostican. Además del subregistro de tales casos, existe el contagio en el hogar y en la comunidad. Por otra parte, haber tenido la enfermedad natural no confiere protección de por vida, por lo cual se debe continuar el programa de vacunación y refuerzos, inclusive en personas quienes hayan tenido tosferina.

Otra medida importante es la vacunación materna, que protege a los niños pequeños que no han comenzado el esquema vacunal y reduce la incidencia y la letalidad de tosferina. La vacunación materna como estrategia segura en la reducción de la incidencia de tosferina escapa al ámbito de esta revisión; sin embargo, existen revisiones recientes del tema y los primeros datos de impacto en Latinoamérica ${ }^{4,33,34,40,41}$.

\section{Recomendaciones del grupo de expertos en vacunación del lactante de SLIPE y ALAPE}

El Grupo de Expertos de SLIPE y ALAPE se reunió para presentar recomendaciones con base en los datos revisados. Estas recomendaciones se resumen en la Tabla 3 y se presentan a continuación. 


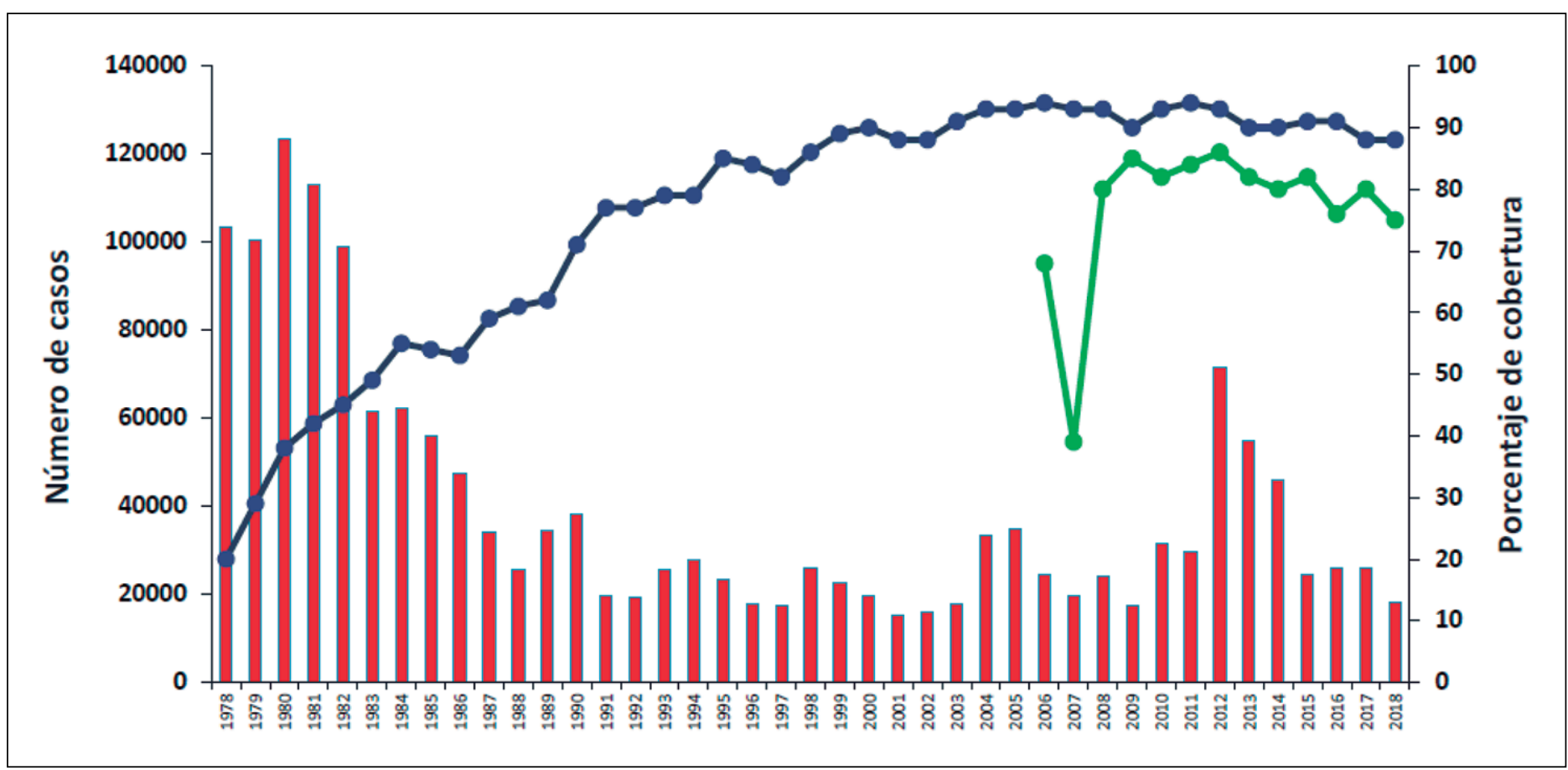

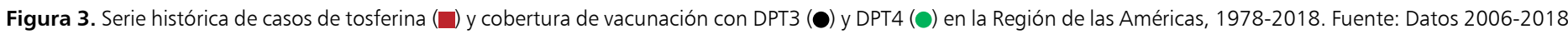
del formulario conjunto para la notificación sobre inmunización de la OPS/OMS y UNICEF, 2019 (JRF por sus siglas en inglés).

Tabla 3. Recomendaciones del Grupo de Expertos de SLIPE y ALAPE para la vacunación contra tosferina

- La prevención de tosferina es prioritaria para el lactante debido al riesgo de complicaciones y muerte

- La vacunación de mujeres embarazadas es también prioritaria, segura y costo-efectiva en la prevención de tosferina en los lactantes menores de 3 meses

- Todo niño debe ser vacunado con la primera dosis entre las 6 y 8 semanas de edad cronológica, independientemente de edad gestacional o peso al nacer

- Garantizar coberturas mínimas de $90 \%$ para la serie primaria, aumentando las coberturas en mujeres embarazadas y en los refuerzos del segundo año y del preescolar

- Actualizar la vacunación del lactante que no tiene la vacunación al día; se recomienda el esquema acelerado con intervalo mínimo de un mes entre las dosis de la serie primaria

- Utilizar, de preferencia, vacunas aP en combinación con otras vacunas, para reducir el número de punciones y mejorar la aceptación por parte de los padres y facilitar la logística de administración de múltiples vacunas

- Actualizar los esquemas de vacunación, incluyendo los refuerzos, preferiblemente antes del reinicio de clases

- Mejorar los sistemas de vigilancia; es ideal que todos los países de la región tengan una definición de caso unificada para tosferina que incluya la clínica, el laboratorio y el nexo epidemiológico, para facilitar la vigilancia y comparación de datos entre países

- Facilitar el acceso a las informaciones epidemiológicas actualizadas por parte de los profesionales de salud

- Aprovechar todas las oportunidades para vacunar; no importa tener la mejor vacuna, si no se administra a tiempo

ALAPE: Asociación Latinoamericana de Pediatría; SLIPE: Sociedad Latinoamericana de Infectología Pediátrica. 
- Es necesario mejorar los sistemas de vigilancia, y deseable que todos los países de la región tengan una definición de caso unificada para tosferina que incluya la clínica, el diagnóstico de laboratorio y el nexo epidemiológico, para que se puedan comparar los datos de los diferentes países;

- El éxito de la vacunación contra tosferina, en parte, es responsable de la poca percepción en la comunidad sobre los riesgos de esta enfermedad, especialmente en los lactantes. Es fundamental que las autoridades de salud faciliten el acceso de los profesionales de salud a datos epidemiológicos actualizados y que las sociedades médicas de cada país actúen en conjunto con las autoridades informando y educando los profesionales de salud y la comunidad sobre los riesgos de tosferina y los beneficios de la vacunación oportuna para alcanzar coberturas más altas y homogéneas.

- Finalmente, hay que buscar todas las oportunidades para vacunar: no importa cuál vacuna tengamos, lo importante es administrarla en forma oportuna.

Agradecimientos. Los autores desean reconocer la colaboración de Humberto López Castillo, MD, PhD, CPH, CMI en la preparación y revisión del manuscrito, incluyendo las tablas, figuras, traducción y revisiones subsiguientes.

\section{Referencias bibliográficas}

1.- Cherry J D. The 112-year odyssey of pertussis and pertussis vaccines-mistakes made and implications for the future. J Pediatric Infect Dis Soc. 2019; 8 (4): 334-41. doi: 10.1093/ jpids/piz005.

2.- Gabutti G, Rota M C. Pertussis: A review of disease epidemiology worldwide and in Italy. Int J Environ Res Public Health. 2012; 9 (12): 4626-38. doi: 10.3390/ijerph9124626.

3.- Folaranmi T, Pinell-McNamara V, Griffith M, Hao Y, Coronado F, Briere E C. Systematic review and meta-analysis of pertussis epidemiology in Latin America and the Caribbean: 1980-2015. Rev Panam Salud Publica. 2017; 41: e102. doi: 10.26633/ RPSP.2017.102.

4.- Gentile A, Bricks L, Avila-Aguero M L, Avila Kfouri R, Torres Torreti J P, UlloaGutierrez R. Pertussis in Latin America and the Hispanic Caribbean: a systematic review. Expert Rev Vaccines. 2019; 18 (8): 829-45. doi: 10.1080/14760584.2019.1643241

5.- Hozbor D, Ulloa-Gutierrez R, Marino C, Wirsing von Konig C H, Tan T, Forsyth K. Pertussis in Latin America: recent epidemiological data presented at the 2017 Global Pertussis Initiative meeting. Vaccine.
2019; 37 (36): 5414-21. doi: 10.1016/j. vaccine.2019.07.007.

6.- Torres Martínez C, Aguilar Velasco M, Álvarez Moreno C, Arbo Sosa A, Ávila Agüero M L, Bonvehi P E, et al. Documento Latinoamericano sobre Vacunación y Servicios de Inmunización durante la Pandemia COVID-19. 2020. https://slipe.org/ web/wp-content/uploads/2020/06/COVID-19. pdf.

7.- Falleiros Arlant L H, de Colsa A, Flores D, Brea J, Avila Aguero M L, Hozbor D F. Pertussis in Latin America: epidemiology and control strategies. Expert Rev Anti Infect Ther. 2014; 12 (10): 1265-75. doi: $10.1586 / 14787210.2014 .948846$.

8.- Edwards K M, Decker M D. Pertussis vaccines. In: Plotkin S A, Orenstein W A, Offit P A, eds. Plotkin's Vaccines. 7 ed. Elsevier; 2018: Cap. 44. pages 711-61.

9.- WHO. Pertussis vaccines: WHO position paper, August 2015-Recommendations. Vaccine. 2016; 34 (12): 1423-5. doi:10.1016/j. vaccine.2015.10.136.

10.- WHO. Pertussis. Consultado el 05 de octubre de 2020 en https://www.who.int/health-topics/ pertussis\#tab=tab_1.

11.- Vaccine-preventable diseases: Whooping cough (pertussis). Consultado el 05 de octubre de 2020 en https://vaccineinformation.org/ whooping-cough/.

12.- Leber A L. Pertussis: relevant species and diagnostic update. Clin Lab Med. 2014; 34 (2): 237-55. doi: 10.1016/j.cll.2014.02.003.

13.- Aquino-Andrade A, Martínez-Leyva G, Merida-Vieyra J, Saltigeral P, Lara A, Domínguez W, et al. Real-time polymerase chain reaction-based detection of Bordetella pertussis in Mexican infants and their contacts: a 3-tear multicenter study. J Pediatr. 2017; 188: 217-23 e1. doi: 10.1016/j.jpeds.2017.05.032.

14.- Ministerio de Salud (Argentina). Coqueluche: recomendaciones y definiciones. Consultado el 05 de octubre de 2020 en http:// www.msal.gob.ar/images/stories/bes/ graficos/0000000473cnt-2013-10_coquelucherecomendaciones-definiciones.pdf.

15.- European Centre for Disease Prevention and Control. Expert Consultation on Pertussis: Meeting Report. Barcelona, Spain: November 20, 2012. Consulado el 05 de octubre de 2020 en https://www.ecdc.europa.eu/sites/default/ files/media/en/publications/Publications/ pertussis-meeting-2012.pdf.

16.- Craig R, Kunkel E, Crowcroft N S, Fitzpatrick M C, de Melker H, Althouse B M, et al. Asymptomatic infection and transmission of pertussis in households: a systematic 
review. Clin Infect Dis. 2020; 70 (1): 152-61. doi:10.1093/cid/ciz531.

17.- Gangarosa E J, Galazka A M, Wolfe C R, Phillips L M, Miller E, Chen R T, et al. Impact of anti-vaccine movements on pertussis control: the untold story. Lancet. 1998; 351 (9099): 35661. doi: 10.1016/s0140-6736(97)04334-1.

18.- Pertussis surveillance in Sweden, twentyone-year report. Folkhsomyndigheten; 2019. Consultado el 05 de octubre de 2020 en http:// www.folkhalsomyndigheten.se/publiceratmaterial/publikationsarkiv/p/pertussissurveillance-in-sweden-twentyone-year-report/.

19.- Klein N P, Bartlett J, Rowhani-Rahbar A, Fireman B, Baxter R. Waning protection after fifth dose of acellular pertussis vaccine in children. N Engl J Med. 2012; 367 (11): 10129. doi:10.1056/NEJMoa1200850.

20.- Zarei S, Jeddi-Tehrani M, Mehdi Akhondi M, Zeraati H, Ferydonfar A A, Nasernia J, et al. Immunogenicity and reactogenicity of two diphtheria-tetanus-whole cell pertussis vaccines in Iranian pre-school children, a randomized controlled trial. Hum Vaccin Immunother. 2013; 9 (6): 1316-22. doi:10.4161/hv.24093.

21.- Desauziers E, Hessel L, Decker M D, Caro J J, Liese J G. Systematic review of the effects of pertussis vaccines in children. Vaccine. 2004; 22 (21-22): 2681-4; author reply 2685. doi:10.1016/j.vaccine.2004.03.004.

22.- Plotkin S A, Cadoz M. The acellular pertussis vaccine trials: an interpretation. Pediatr Infect Dis J. 1997; 16 (5): 508-17; discussion 517-9. doi:10.1097/00006454-199705000-00011.

23.- Center for Biologics Evaluation and Research. Guidance for industry: providing clinical evidence of effectiveness for human drug and biological products. U S Dept of Health and Human Services, Food and Drug Administration, Center for Drug Evaluation and Research; 1998. Consultado el 05 de octubre de 2020 en http://purl.access.gpo.gov/GPO/ LPS119052.

24.- Decker M D, Edwards K M, Steinhoff M C, Rennels M B, Pichichero M E, Englund J A, et al. Comparison of 13 acellular pertussis vaccines: adverse reactions. Pediatrics. 1995; 96 (3 Pt 2): 557-66. PMID: 7659476.

25.- Zhang L, Prietsch S O, Axelsson I, Halperin S A. Acellular vaccines for preventing whooping cough in children. Cochrane Database Syst Rev.
2014; (9): CD001478. doi: 10.1002/14651858. CD001478.pub6.

26.- Le Saux N, Barrowman N J, Moore D L, Whiting S, Scheifele D, Halperin S and; for Members of the Canadian Paediatric Society/ Health Canada Immunization Monitoring Program-Active (IMPACT). Decrease in hospital admissions for febrile seizures and reports of hypotonic-hyporesponsive episodes presenting to hospital emergency departments since switching to acellular pertussis vaccine in Canada: a report from IMPACT. Pediatrics. 2003; 112 (5): e348. doi:10.1542/ peds.112.5.e348.

27.- Hawken S, Manuel D G, Deeks S L, Kwong J C, Crowcroft N S, Wilson K. Underestimating the safety benefits of a new vaccine: the impact of acellular pertussis vaccine versus whole-cell pertussis vaccine on health services utilization. Am J Epidemiol. 2012; 176 (11): 1035-42. doi: 10.1093/aje/kws167.

28.- Chit A, Zivaripiran H, Shin T, Lee J K H, Tomovici A, Macina D, et al. Acellular pertussis vaccines effectiveness over time: a systematic review, meta-analysis and modeling study. PLoS One. 2018; 13 (6): e0197970. doi: 10.1371/journal.pone.0197970.

29.- Zerbo O, Bartlett J, Goddard K, Fireman B, Lewis E, Klein N P. Acellular pertussis vaccine effectiveness over time. Pediatrics. 2019; 144 (1): e20183466. doi:10.1542/peds.2018-3466.

30.- Korkmaz H A, Aydin A, Unal B. Comparison of acellular pertussis-tetanus-diphtheria vaccines and whole-cell pertussis-tetanusdiphtheria vaccines in infancy. Paediatr Int Child Health. 2014; 34 (3): 198-202. doi: 10.1179/2046905513Y.0000000110

31.- Patterson J, Kagina B M, Gold M, Hussey G D, Muloiwa R. Comparison of adverse events following immunisation with acellular and whole-cell pertussis vaccines: a systematic review. Vaccine. 2018; 36 (40): 6007-16. doi:10.1016/j.vaccine.2018.08.022.

32.- WHO. Reducing pain at the time of vaccination: WHO position paper, September 2015-Recommendations. Vaccine. 2016; 34 (32) :3629-30. doi: 10.1016/j vaccine.2015.11.005.

33.- Ministerio de Salud (Argentina). Coberturas de vacunación por jurisdicción 2009-2019. Consultado el 05 de octubre de 2020 en http:// bancos.salud.gob.ar/recurso/coberturas-devacunacion-por-jurisdiccion-2009-2019.

34.- Gentile A, Juarez M D V, Lucion M F, Martínez A C, Romanin V, Areso S, et al. Bordetella pertussis (Bp) disease: Before (2003-2011) and after (2013-2016) maternal immunization strategy in a pediatric hospital. Vaccine. 2018; 36 (11): 1375-80. doi: 10.1016/j. vaccine.2018.01.091.

35.- Romanin V, Acosta A M, Juarez M D V, Biere E, Sanchez S M, Lopez Cordova B, et al. Maternal vaccination in Argentina: tetanus, diphtheria, and acellular pertussis vaccine effectiveness during pregnancy in preventing pertussis in infants $<2$ months of age. Clin Infect Dis. 2020; 70 (3): 380-7. doi:10.1093/ $\mathrm{cid} / \mathrm{ciz} 217$.

36.- Ministério da Saúde do Brasil. Diário Oficial da União. 17 de julio de 2019.

37.- WHO. WHO vaccine-preventable diseases: monitoring system. 2020 global summary (Brazil). Consultado el 5 de octubre de 2020 en https://apps.who.int/immunization_monitoring/ globalsummary/countries?countrycriteria $\% 5 \mathrm{Bc}$ ountry $\% 5 \mathrm{D} \% 5 \mathrm{~B} \% 5 \mathrm{D}=\mathrm{BRA} \&$ commit $=\mathrm{OK}$.

38.- Olivera I, Grau C, Dibarboure H, Torres J P, Mieres G, Lazarov L, et al. Valuing the cost of improving Chilean primary vaccination: a cost minimization analysis of a hexavalent vaccine. BMC Health Serv Res. 2020; 20 (1): 295. doi: 10.1186/s12913-020-05115-7.

39.- O’Ryan M, Calvo A E, Espinoza M, Vega N, Lagomarcino A J, López Castillo H, et al. Parent reported outcomes to measure satisfaction, acceptability, and daily life impact after vaccination with whole-cell and acellular pertussis vaccine in Chile. Vaccine. 2020; 38 (43): 6704-13. doi: 10.1016/j. vaccine.2020.08.046.

40.- Villena R, Vidal P, Carrillo F, Salinas M. Vacunacion con pertussis en el embarazo: Una estrategia segura y efectiva para proteger al lactante menor. Rev Chil Pediatr. 2017; 88 (3): 318-23. doi: 10.4067/S037041062017000300002.

41.- Fernandes E G, Sato A P S, Vaz-de-Lima L R A, Rodrigues M, Leite D, de Brito C A, et al. The effectiveness of maternal pertussis vaccination in protecting newborn infants in Brazil: A casecontrol study. Vaccine. 2019; 37 (36): 5481-4. doi: 10.1016/j.vaccine.2019.03.049. 